\title{
Managing back pain and osteoarthritis without paracetamol
}

\author{
Physical treatments are the way forward
}

\author{
Christian Mallen NIHR research professor in general practice, Elaine Hay professor of community \\ rheumatology
}

Arthritis Research UK Primary Care Centre, Research Institute for Primary Care and Health Sciences, Keele University, Keele ST5 5BG, UK

Paracetamol (acetaminophen) is a drug we are all familiar with. It is cheap, readily available over the counter, and is common place in family medicine cabinets across the world. We rely on it in the middle of the night to settle childhood fevers, and it is usually our first choice drug for a wide range of painful musculoskeletal disorders, including osteoarthritis and spinal pain. Yet new evidence in a linked paper by Machado and colleagues (doi:10.1136/bmj.h1225) casts doubt on the efficacy and safety of paracetamol and questions its place as our first choice analgesic. ${ }^{1}$

In their systematic review and meta-analysis examining the efficacy and safety of paracetamol for spinal pain and osteoarthritis, Machado and colleagues synthesised evidence from 13 randomised controlled trials. ${ }^{1}$ They report that there is "high quality" evidence that paracetamol is ineffective for reducing pain intensity and disability in patients with low back pain and "high quality" evidence that paracetamol produces a significant but not clinically important effect on pain and disability in patients with osteoarthritis. While adverse events were reported at a similar level to that seen with placebo, patients taking paracetamol were nearly four times more likely to have abnormal results on liver function tests. These findings encourage us to reassess the role of paracetamol as the key analgesic in managing osteoarthritis and spinal pain.

Some of these findings are not surprising. The recently revised guidelines from the National Institute for Health and Care Excellence on the management of osteoarthritis caused controversy when draft guidance advised that paracetamol should not be routinely offered to patients as it might not be effective and was potentially associated with important side effects when used at high doses for a prolonged period of time. This decision was reversed in the full guideline after concerns from a range of stakeholders that this change in guidance might dramatically increase the prescribing of other potentially harmful drugs including non-steroidal anti-inflammatory drugs and opioids. ${ }^{2}$ The Medicine and Healthcare Products Regulatory Agency is currently reviewing the safety of over the counter analgesics, and the findings might also influence prescribing guidance. The new evidence provided by Machado and colleagues re-opens the debate, leaving patients and clinicians wondering what is left that can help to manage these common, painful, and highly disabling conditions.

There are alternative safe and effective treatments available, although evidence suggests that they are used inconsistently. Over the past 10 years prescriptions for topical NSAIDs, such as ibuprofen, have more than doubled in the UK. ${ }^{3}$ Topical NSAIDs (such as ibuprofen) are available over the counter, are as effective as oral NSAIDs for knee osteoarthritis, and are associated with fewer side effects. ${ }^{4}$ They are also popular with patients, who prefer using topical NSAIDs for mild, local, and transient pain. ${ }^{5}$ Oral NSAIDs are effective but are best limited to short courses in patients without contraindications-making them unsuitable for many, especially older, patients with multiple health conditions. The evidence supporting the use of opioids for osteoarthritis and spinal pain is limited, and while the UK has yet to see the dramatic increase in prescribing observed in the United States, a reduction in the use of paracetamol could result in increased prescribing rates and a new range of associated problems, especially in high risk groups. Ongoing and ever increasing concerns about pharmacological management of musculoskeletal pain highlights the importance of non-pharmacological options, which form the cornerstone of self management of spinal pain and osteoarthritis. NICE recommends that all patients with osteoarthritis should receive written information with advice about maintaining or increasing physical activity and optimising weight (if appropriate); exercise, manual therapy, acupuncture, and psychological support are also recommended for those with back pain. While the effectiveness of exercise for both osteoarthritis ${ }^{6}$ and spinal pain ${ }^{7}$ is established, we know that uptake of, and adherence to, exercise is poor. ${ }^{8}$ Health professionals, particularly in primary care, where most of these patients are managed, have an important role to play in supporting individuals to exercise. Physiotherapists are key professionals to offer expert advice and support in this regard, but timely access to physiotherapy services is becoming increasingly limited in the NHS, 
constraining the usefulness of this evidence based and safe treatment. Trials of exercise based interventions for musculoskeletal pain and osteoarthritis typically show the same response pattern of a short term reduction in levels of pain and disability with demonstrable clinical benefit tailing off over 12-24 months. Maintaining the benefit of exercise is a current research priority, with approaches to improve adherence being a key target.

Spinal pain and osteoarthritis are common reasons for seeking healthcare, accounting for around 10-15\% of all consultations with a general practitioner. Management of these patients can be challenging and is compounded by emerging evidence about the safety and effectiveness of commonly used drugs that conflicts with established clinical practice and published guidelines. Changing behaviour of doctors and their patients is notoriously difficult, but the findings of Machado and colleagues emphasise that the time has come to shift our attention away from tablets as the default option for managing chronic musculoskeletal pain. Non-pharmacological treatments work, are safe, and have benefits that reach beyond the musculoskeletal system. The challenge for patients, providers of healthcare, and policy makers is how to ensure access to, uptake of, and adherence to these self management approaches.
Competing interests: We have read and understood the BMJ Group policy on declaration of interests and have no interest to declare.

Provenance and peer review: Commissioned; not externally peer reviewed.

1 Machado GC, Maher CG, Ferreira PH, et al. Efficacy and safety of paracetamol for spinal pain and osteoarthritis: systematic review and meta-analysis of randomised placebo controlled trials. BMJ 2015;350:h1225.

2 National Institute for Health and Care Excellence. Osteoarthritis: care and management in adults. NICE, 2014. www.nice.org.uk/guidance/cg177.

3 Bedson J, Belcher J, Martino OI, et al. The effectiveness of national guidance in changing analgesic prescribing in primary care from 2002 to 2009: an observational database study. Eur J Pain 2013;17:434-43.

4 Underwood M, Ashby D, Cross P, et al. Advice to use topical or oral ibuprofen for chronic knee pain in older people: randomised controlled trial and patient preference study. BMJ 2008;336:138-42.

5 Carnes D, Anwer Y, Underwood M, et al. Influences on older people's decision making regarding choice of topical or oral NSAIDs for knee pain: qualitative study. BMJ 2008;336:142-5.

6 Uthman OA, van der Windt DA, Jordan JL, et al. Exercise for lower limb osteoarthritis: systematic review incorporating trial sequential analysis and network meta-analysis. BMJ 2013;347:f5555.

7 Hayden JA, van Tulder MW, Malmivaara AV, et al. Meta-analysis: exercise therapy for nonspecific low back pain. Ann Intern Med 2005;142:765-75

8 Jordan JL, Holden MA, Mason EE, et al. Interventions to improve adherence to exercise for chronic musculoskeletal pain in adults. Cochrane Database Syst Rev 2010;1:CD005956.

Cite this as: BMJ 2015;350:h1352

(c) BMJ Publishing Group Ltd 2015 